Т.Е. Привалова ${ }^{1,2}$, О.В. Суровцева ${ }^{1}$, Д.В. Андриянов ${ }^{1}$

1 Российский национальный исследовательский медицинский университет им. Н.И. Пирогова, Москва, Российская Федерация

2 НИИ педиатрии и охраны здоровья детей ЦКБ РАН Министерства науки и высшего образования РФ, Москва, Российская Федерация

\title{
Преимущества гендерно-нейтральной
} стратегии вакцинации для профилактики ВПЧ-ассоциированных заболеваний и искоренения ВПЧ-инфекции в целом. Актуальное состояние вакцинации против ВПЧ в мире

Автор, ответственный за переписку:

Суровцева Ольга Викторовна, ординатор кафедры факультетской педиатрии педиатрического факультета ФГАОУ ВО “Российский национальный исследовательский медицинский университет им. Н.И. Пирогова" Министерства здравоохранения Российской Федерации Адрес: 117997, Москва, ул. Островитянова, д. 1, e-mail: dr.surovtseva@yandex.ru

Папилломавирусная инфекция (ВПЧ-инфекция) по распространенности занимает лидирующее место среди инфекций, передающихся половым путем. ВПЧ-инфекция и ассоциированные с ней заболевания, в том числе онкологические, остаются одной из глобальных проблем современности. В литературном обзоре представлены актуальные данные зарубежных исследований и отечественных работ о состоянии вакцинации против ВПЧ в мире, об эффективности применения современных вакцин для профилактики ВПЧ-ассоциированных заболеваний, об используемых в настоящее время стратегиях вакцинации и их эффективности. Целью статьи является определение преимуществ гендерно-нейтральной стратегии вакцинации перед приоритетно используемой стратегией вакцинации только девочек при профилактике ВПЧ-ассоциированных заболеваний, в том числе онкологических, а также для искоренения инфекции ВПЧ в целом.

Ключевые слова: вирус папилломы человека, ВПЧ-ассоциированные заболевания, вакцинация, стратегии вакцинации, гендерно-нейтральная вакцинация

Для цитирования: Привалова Т.Е., Суровцева О.В., Андриянов Д.В. Преимущества гендерно-нейтральной стратегии вакцинации для профилактики ВПЧ-ассоциированных заболеваний и искоренения ВПЧ-инфекции в целом. Актуальное состояние вакцинации против ВПЧ в мире. Педиатрическая фармакология. 2021;18(3):239-244. doi: 10.15690/pf.v18i3.2285

\section{ОБОСНОВАНИЕ}

Вирус папилломы человека (ВПЧ, HPV - англ. human papillomavirus) - неинкапсулированный ДНКвирус семейства Papillomaviridae, передающийся половым путем, обладающий тропизмом к клеткам плоского эпителия [1-3]. Вирус проникает в эпителий через повреждения кожи или слизистой оболочки, поражает базальные стволовые клетки и при помощи механизмов активного и пассивного уклонения от иммунной системы длительно персистирует в организме. Жизненный цикл ВПЧ полностью внутриэпителиальный, без виремии, лизиса клеток или воспаления, таким образом, вирус находится вне поля зрения как адаптивного, так и врожденного иммунного ответа [4-6]. Выявлено более 200 типов ВПЧ, которые группируются в пределах рода: альфа (a) Alphapapillomavirus, бета $(\beta)-$ Betapapillomavirus, гамма $(\gamma)$ - Gammapapillomavirus, $\mathrm{Mu}(\mu)$ - Mupapillomavirus и Nu (v) - Nupapillomavirus [3-9]. ВПЧ может вызывать стойкие субклинические инфекции, а также клиниче- ски выраженные - как у иммунокомпрометированных, так и у иммунокомпетентных организмов [5, 10]. Вирусы рода альфа подразделяются на типы в зависимости от их онкогенного потенциала: ВПЧ высокого (hrHPV) и низкого риска (IrHPV). ВПЧ высокого онкогенного потенциала может привести к раку шейки матки (РШМ) и ВПЧ-ассоциированному раку других локализаций: вульвы, влагалища, головы и шеи у женщин, полового члена, ануса, головы и шеи - у мужчин. Предраковые и раковые поражения шейки матки, мужских и женских аногенитальных зон и ротоглотки чаще всего вызываются 16-м и 18-м подтипами ВПЧ, однако подтипы 31, 33, 35, 45, 52 и 58-й также относятся к группе ВПч Высокого онкогенного риска [1, 3, 11-17]. ВПЧ низкого онкогенного потенциала (HPV 6-го и 11-го; 1, 2, 3, 4, 10, 27 и 57-го подтипов) вызывают широкий спектр других ВПЧ-ассоциированных заболеваний: аногенитальные кондиломы у мужчин и женщин, респираторный рецидивирующий папилломатоз - у детей раннего возраста. Они не считаются значимыми триггерами злокачествен- 
ной трансформации. Однако в некоторых публикациях 6-й и 11-й подтипы относят к ВПЧ, обладающим хоть и низким, но онкогенным потенциалом $[1-4,8,18]$.

Точные сведения о глобальной распространенности инфекции ВПЧ и ассоциированных заболеваний в открытых источниках отсутствуют, так как не во всех странах проводят учет и регистрацию клинических проявлений, а также расчет статистических показателей (в том числе в России). Наиболее актуальные и полные сведения по распространенности ВПЧ-ассоциированных раков, находящиеся в открытом доступе, представлены в статистическом отчете Национального института рака США. Во всем мире бремя рака, связанного с ВПЧ, велико. Около 5\% всех онкологических заболеваний в мире связано с ВПЧ высокого риска [19]. По данным Международного агентства по изучению рака (МАИР, IARC), среди инфекционных агентов, связанных с раком различной локализации, ВПЧ занимает доминирующее место у женщин $(35,9-75,9 \%)$ и варьирует в широком диапазоне значений у мужчин $(1,3-17,8 \%)$ в зависимости от континента $[20,21]$. Для снижения бремени ВПЧассоциированных заболеваний, в том числе рака, разработана специфическая профилактика ВПЧ-инфекции, а именно вакцинация [22-33].

\section{Цель работы}

Изучить зарубежный опыт, показывающий преимущества гендерно-нейтральной стратегии вакцинации перед приоритетно-используемой стратегией вакцинации только девочек при профилактике ВПчассоциированных заболеваний, в том числе онкологических, а также для искоренения инфекции ВПЧ в целом.

\section{АКТУАЛЬНОЕ СОСТОЯНИЕ ВАКЦИНАЦИИ ПРОТИВ ВПЧ В МИРЕ}

В настоящее время в мире доступны три профилактических вакцины против ВПЧ: двухвалентная (против ВПЧ 16-го и 18-го типов), четырехвалентная (против ВПЧ 6, 11, 16 и 18-го типов) и девятивалентная (против ВПЧ 6 , $11,16,18,31,33,45,52$ и 58-го типов). В России на данный момент зарегистрировано два препарата - двухва- лентная и четырехвалентная вакцины. Данные вакцины субъединичные. В их составе рекомбинантные вирусоподобные частицы (VLP), не содержащие ДНК, полученные путем экзогенной экспрессии главного капсидного белка (L1) BПЧ в дрожжах (четырехвалентная) и в клетках насекомых (двухвалентная). Все вакцины прошли крупные рандомизированные контролируемые двойные слепые испытания III фазы (РКИ) с участием молодых женщин в возрасте 15-26 лет, где продемонстрировали эффективность > 90\% против заболеваний, связанных с типом вакцины против ВПЧ у лиц, ранее не подвергавшихся вакцинации против ВПЧ. Введение вакцины осуществляется внутримышечно, двух- или трехкратно в течение 6-месячного периода. Поскольку ВПЧ в основном передается при половом контакте, рекомендовано проведение вакцинации в подростковом возрасте до начала половой жизни. Вакцины не влияют на уже существующую инфекцию ВПЧ [22-29]. После вакцинации отмечается сильный стабильный ответ антител продолжительностью более десяти лет [30]. Даже однократная доза эффективна для предотвращения развития инфекции и предраковой неоплазии [23]. Статистические данные последних лет из разных стран с разным уровнем охвата вакцинацией против ВПЧ и разными стратегиями внедрения показывают, что использование вакцин против ВПЧ эффективно для предотвращения инфекций и заболеваний, связанных с конкретными генотипами ВПЧ [31].

Принимая во внимание социальное и экономическое бремя ВПЧ-ассоциированных онкологических заболеваний, Всемирная организация здравоохранения (ВОЗ) рекомендует включить вакцинацию против ВПЧ в национальные программы иммунизации, так как вакцинопрофилактика является наиболее доступным, эффективным и безопасным способом первичной профилактики ВПчассоциированных раков и других ВПЧ-ассоциированных заболеваний у мужчин, женщин и детей раннего возраста, а также папилломавирусной инфекции (ПВИ) в целом $[22,32,34]$. Программы вакцинации против ВПЧ успешно внедрены во многих странах мира, причем их количество ежегодно растет. К концу 2019 г. рутинная вакцинация

Tatiana E. Privalova ${ }^{1,2}$, Olga V. Surovtseva ${ }^{1}$, Dmitry V. Andriyanov ${ }^{1}$

${ }^{1}$ Pirogov Russian National Research Medical University, Moscow, Russian Federation

${ }^{2}$ Research Institute of Pediatrics and Children's Health in "Central Clinical Hospital of the Russian Academy of Sciences", Moscow, Russian Federation

\section{Benefits of Gender-Neutral Vaccination Strategy for the Prevention of HPV-Associated Diseases and the Eradication of HPV Infection in General. Current State of HPV Vaccination in the World}

Papillomavirus infection (HPV infection) is the leading sexually transmitted infection in prevalence. HPV infection and associated diseases, including cancer, remain one of the global problems of our time. This literature review presents relevant data from foreign studies and domestic works on the state of HPV vaccination in the world, the modern vaccines efficacy for the HPV-associated diseases prevention, current vaccination strategies and their efficacy as well. The aim of the article is to determine the advantages of using gender-neutral vaccination strategy over priority-used vaccination strategy for girls only in the prevention of HPV-associated diseases, including cancer, as well as for eradicating HPV infection in general.

Keywords: human papillomavirus, HPV-associated diseases, vaccination, vaccination strategies, gender-neutral vaccination

For citation: Privalova Tatiana E., Surovtseva Olga V., Andriyanov Dmitry V. Benefits of Gender-Neutral Vaccination Strategy for the Prevention of HPV-Associated Diseases and the Eradication of HPV Infection in General. Current State of HPV Vaccination in the World. Pediatricheskaya farmakologiya - Pediatric pharmacology. 2021;18(3):239-244. doi: 10.15690/pf.v18i3.2285 
против ВПЧ проводилась в 106 странах, к середине 2020 г. вакцинация против ВПЧ внедрена в национальные календари 131 страны, треть государств в их числе используют принцип гендерно-нейтральной вакцинации. [31-37]. В настоящее время вакцинация против ВПч не включена в национальный календарь профилактических прививок в России, однако внедрена в региональные программы иммунизации и осуществляется в виде пилотных проектов в некоторых субъектах (Московская область, Свердловская область, Санкт-Петербург, ХантыМансийский автономный округ, Якутск, Новосибирск, Смоленская область и др.) [32, 38-40].

На сегодняшний день вакцинация против ВПЧ является широко распространенной политикой здравоохранения во всем мире, однако глобальный показатель охвата последней дозой вакцины в настоящее время оценивается на уровне 15\%. Это обусловлено тем, что она еще не введена в рутинный график вакцинации большого числа крупных стран (включая Российскую Федерацию), а во многих странах ею охвачена недостаточная доля населения, в том числе по причине применения стратегии вакцинации только девочек. Преимущественно охват вакцинацией против ВПЧ только девочек варьирует от низкого (20-50\% во Франции и США) до умеренного (50-60\% в Нидерландах, 70-80\% - в Финляндии, Швеции, Великобритании) [35, 41-45]. Причем в связи с пандемией COVID-19 во многих странах мира кампании по вакцинации были приостановлены или произошло снижение охвата вакцинацией, в том числе против ВПч [34, 36, 37].

Защита от папилломавирусной инфекции (ПВИ) осуществляется при формировании иммунитета непосредственно среди вакцинированного населения, а также посредством формирования коллективного иммунитета первого и второго порядков. Для создания коллективной защиты значение имеют как тип HPV (несколько вакцинных штаммов) или индуцированная вакцинацией перекрестная защита (к штаммам, не содержащимся в вакцине), так и выбранная стратегия вакцинации [4648]. Распространенность ВПч 16-го типа в популяциях повсеместно как минимум в два раза выше, чем распространенность ВПЧ 18, 31, 33, 45-го типов [49-51]. При наблюдении за программами и моделями вакцинации установлено, что снижение распространенности ВПЧ 16-го типа среди невакцинированного населения, основанное на эффекте коллективного иммунитета, требует очень высокого охвата вакцинацией [49-52]. В систематическом обзоре было показано значительное сокращение аногенитальных бородавок (вызываемых ВПч 6-го и 11-го типов) у молодых мужчин в первые 4 года после внедрения программ вакцинации только девочек четырехвалентной вакциной с высоким охватом ( $\geq 50 \%$ ), но при этом отсутствие признаков воздействия на популяцию в странах с низким охватом вакцинацией $(<50 \%)$. Это говорит о том, что при условии достижения достаточного охвата вакцинацией (умеренного или высокого) против ВПЧ только девочек вирусы низкого онкогенного риска можно контролировать с помощью вакцинации, так как формируется коллективный иммунитет, что позволяет защитить также невакцинированных мужчин и/или женщин от ВПЧ 6-го и 11-го типов [49, 50, 53-56]. Например, страны, использующие четырехвалентную вакцину против ВПч, продемонстрировали снижение числа диагнозов генитальных бородавок в целевых когортах и в нецелевых когортах мужчин, что является свидетельством формирования протективного эффекта первого порядка [31]. Данные эпиднадзора в Австралии указывают также на снижение заболеваемости рецидивирующим респираторным папилломатозом у подростков (заболевание обусловлено вертикальной передачей инфекции HPV 6-го и 11-го типов от инфицированной матери младенцу), вероятно, обусловленной низкой распространенностью HPV 6-го и 11-го типов у женщин после вакцинации в этой стране [57]. Недавно была описана перекрестная защита против ВПЧ 31-го и 33-го типов после вакцинации только девочек с высоким охватом или гендерно-нейтральной вакцинации с низким и средним охватом двухвалентной вакциной $[56,58]$. Таким образом, даже при гендернонейтральной стратегии вакцинации ВПЧ 16-го типа было бы трудно контролировать с помощью охвата вакцинацией против ВПЧ 16-го и 18-го типов от низкого до среднего [49-51].

Из-за повышенных ожиданий относительно охвата вакцинацией только девочек и неполного учета общего бремени ВПЧ стратегия вакцинации только девочек доминировала. Вакцинация девочек против ВПЧ с очень высоким (> 90\%) охватом может привести к искоренению онкогенных ВПЧ посредством коллективной защиты, но такого высокого охвата добиться трудно. В Шотландии, где проводились программы вакцинации только девочек против ВПч с высоким охватом (90 и 70\%), сообщили о значительных эффектах коллективной защиты против ВПч 6-го и 11-го типов, а также о коллективной защите против ВПЧ 16-го и 18-го типов женщин $[50,56]$. Искоренение ВПч 16-го и 18-го типов с помощью вакцинации только девочек требует генерации сильного протективного эффекта второго порядка против данных типов ВПч среди невакцинированных девочек и невакцинированных мальчиков, что требует исключительно высокого (> 90\%) охвата вакцинацией [49]. В глобальном масштабе нынешний уровень охвата вакцинацией от низкого до среднего не обеспечивает коллективной защиты при программе вакцинации только девочек [35, 41, 42]. Ключевым вопросом для решения данной проблемы является внедрение расширений программы с усилением охвата вакцинации девочек и молодых женщин (улучшение охвата и/или расширение целевых возрастных диапазонов) и/или распространение программы вакцинации против ВПч на мальчиков. Например, в США увеличение охвата вакцинацией против ВПЧ за 2017-2018 гг. было связано только с увеличением числа вакцинированных мужчин [44].

\section{ПРЕИМУЩЕСТВА ГЕНДЕРНО-НЕЙТРАЛЬНОГО ПОДХОДА К ВАКЦИНАЦИИ}

Недавние модели показали, что гендерно-нейтральный подход к вакцинации более эффективен там, где охват вакцинацией умеренный или низкий [51, 52]. При вакцинации только девочек мужчины получают косвенную пользу посредством коллективной защиты, но остаются в группе риска рака, связанного с ВПЧ. Даже при высоком уровне вакцинации девочек преимущества вакцинации мальчиков обусловлены профилактикой анальных карцином, что подчеркивает важность усилий по профилактике ВПЧ для мужчин, практикующих половые контакты с мужчинами. Проведенная работа по моделированию оценила прямую пользу вакцинации мужчин и предполагает, что вакцинация с учетом гендерного фактора является рентабельной [45]. Гендернонейтральная вакцинация не только обеспечивает защиту мальчиков, но и дает возможность усилить коллективный иммунитет [46]. Коллективный эффект, достигаемый с помощью гендерно-нейтральной стратегии вакцина- 
ции, способен быстро заменить более низкую эффективность вакцины против типов ВПЧ с перекрестной защитой. При низком или умеренном охвате вакцинацией высокая эффективность двухвалентной вакцины против HPV 16, 18 и 45-го типов вместе с быстрым коллективным эффектом против HPV 18-го типа и HPV 31, 33 и 35-го типов обеспечивают сравнимую общую защитную эффективность против шести онкогенных типов $\operatorname{HPV}(16,18,31,33,35$ и 45-го) при применении нейтральной в гендерном отношении стратегии вакцинации [48]. Несмотря на то, что гендерно-нейтральная стратегия повысила эффективность вакцинации против ВПЧ с перекрестно защищенными типами ВПЧ с низким и средним охватом, высокий охват среди мужчин, по-видимому, является ключом к обеспечению существенной пользы для общественного здравоохранения, а также для невакцинированных женщин (генерация протективного эффекта второго порядка). Гендернонейтральная вакцинация подростков препубертатного периода вызывает значительные эффекты коллективной защиты и усиливает общую защитную эффективность против ряда типов ВПЧ высокого риска, “покрытых" вакциной, когда применяется охват вакцинацией от низкого до среднего [58].

Конечные результаты нейтральной с гендерной точки зрения профилактической вакцинации против ВПЧ вполне реальны. Страновая ликвидация онкогенных типов ВПЧ и элиминация болезней, ассоциированных с ВПЧ, достижимы уже в обозримом будущем благодаря применению нейтральной в гендерном отношении стратегии вакцинации против ВПч - даже при лишь умеренном охвате. Кроме того, гендерно-нейтральная вакцинация лучше подходит для искоренения онкогенных ВПЧ [46].

\section{ЗАКЛЮЧЕНИЕ}

В заключение считаем необходимым сказать, что защита женщин от заболеваний, вызванных ВПЧ, не должна быть единственной целью общественного здравоохранения при принятии решения о проведении вакцинации против ВПЧ, поскольку мужчины также подвержены риску возникновения заболеваний (в том числе рака), которые можно предотвратить с помощью вакцинации. Если вакцинация только девочек не устранит инфекцию ВПЧ вакцинного типа среди населения, то мужчины останутся уязвимыми для предотвратимых с помощью вакцинации форм рака, особенно имеющие половые контакты с мужчинами, которые в большей степени страдают от рака, вызванного ВПЧ. В долгосрочной перспективе целью является ликвидация ВПЧ-

\section{СПИСОК ЛИТЕРАТУРЫ / REFERENCES}

1. Harden ME, Munger K. Human papillomavirus molecular biology. Mutat Res Rev Mutat Res. 2017;772:3-12. doi: 10.1016/j.mrrev.2016.07.002

2. Bacaj P, Burch D. Human Papillomavirus Infection of the Skin. Arch Pathol Lab Med. 2018;142(6):700-705. doi: 10.5858/arpa.2017-0572-RA

3. Luria L, Cardoza-Favarato G. Human Papillomavirus. In: StatPearls. Treasure Island (FL): StatPearls Publishing; January 24, 2021. Available online: https://www.statpearls.com/ArticleLibrary/ viewarticle/23034. Accessed on July 6, 2021.

4. Steinbach A, Riemer AB. Immune evasion mechanisms of human papillomavirus: An update. Int J Cancer. 2018;142(2):224229. doi: 10.1002/ijc.31027

5. Egawa N, Egawa K, Griffin H, Doorbar J. Human Papillomaviruses; Epithelial Tropisms, and the Development of Neoplasia. Viruses. 2015;7(7):3863-3890. doi: 10.3390/v7072802 ассоциированных заболеваний и элиминации ВПЧинфекции в целом. При использовании вакцинации только девочек необходимо поддержание очень высокого (более 90\%) уровня охвата вакцинацией, что труднодостижимо. Однако при использовании гендерно-нейтральной стратегии (включение мальчиков в программы вакцинации девочек) достаточно поддержание умеренного охвата вакцинацией (70-80\%).

\section{ВКЛАД АВТОРОВ}

Авторы данной статьи внесли равноценный вклад в концепцию и дизайн работы, написание текста и критический пересмотр его содержания, утверждение окончательного варианта статьи для публикации. В равной степени авторы несут ответственность за все аспекты работы, надлежащее изучение и решение вопросов, связанных с достоверностью данных и целостностью всех частей статьи.

\section{AUTHORS' CONTRIBUTION}

The authors of this article have performed equal contribution in the article concept and design, writing, recension, final approval of the article before publication. Equally, all authors are responsible for all aspects of the work, proper studying and resolving issues related to the data reliability and the article complexity.

\section{ИСТОЧНИК ФИНАНСИРОВАНИЯ}

\section{Отсутствует.}

\section{FINANCING SOURCE}

Not specified.

\section{РАСКРЫТИЕ ИНТЕРЕСОВ}

Т.Е. Привалова - получение гонораров от компаний ООО "МСД Фармасьютикалс", ООО “Бионорика", ООО "Нутриция". Остальные авторы статьи подтвердили отсутствие конфликта интересов, о которых необходимо заявить.

\section{DISCLOSURE OF INTEREST}

Tatiana E. Privalova - receiving fees from pharmaceutical companies "MSD Pharmaceuticals", "Bionorica”, "Nutricia”.

Other authors confirmed the absence of a reportable conflict of interests.

ORCID
T.E. Привалова
https://orcid.org/0000-0003-4680-2925
O.В. Суровцева
https://orcid.org/0000-0002-5774-6984
Д.В. Андриянов
https://orcid.org/0000-0003-3494-6614

6. Bravo IG, Félez-Sánchez M. Papillomaviruses: Viral evolution, cancer and evolutionary medicine. Evol Med Public Health. 2015;2015(1):32-51. doi: 10.1093/emph/eov003

7. Nunes EM, Talpe-Nunes V, Sichero L. Epidemiology and biology of cutaneous human papillomavirus. Clinics (Sao Paulo). 2018;73(Suppl 1):e489s. doi: 10.6061/clinics/2018/e489s

8. Egawa N, Doorbar J. The low-risk papillomaviruses. Virus Res. 2017;231:119-127. doi: 10.1016/j.virusres.2016.12.017

9. Papillomavirus Episteme (PaVE): A resource of the Bioinformatics and Computational Biosciences Branch at the National Institute of Allergy and Infectious Diseases (NIAID) Office of Cyber Infrastructure and Computational Biology. Available online: https://pave.niaid.nih. gov. Accessed on March 30, 2021.

10. Bedell SL, Goldstein LS, Goldstein AR, Goldstein AT. Cervical Cancer Screening: Past, Present, and Future. Sex Med Rev. 2020;8(1):28-37. doi: 10.1016/j.sxmr.2019.09.005 
11. Kobayashi K, Hisamatsu K, Suzui N, Hara A, Tomita H, Miyazaki T. A Review of HPV-Related Head and Neck Cancer. J Clin Med. 2018;7(9):241. doi: 10.3390/jcm7090241

12. Timbang MR, Sim MW, Bewley AF, Farwell DG, Mantravadi A, Moore MG. HPV-related oropharyngeal cancer: a review on burden of the disease and opportunities for prevention and early detection. Hum Vaccin Immunother. 2019;15(7-8):1920-1928. doi: 10.1080/21645515.2019.1600985

13. Spence T, Bruce J, Yip KW, Liu FF. HPV Associated Head and Neck Cancer. Cancers (Basel). 2016;8(8):75. doi: 10.3390/cancers8080075 14. Bratman SV, Bruce JP, O'Sullivan B, et al. Human Papillomavirus Genotype Association With Survival in Head and Neck Squamous Cell Carcinoma. JAMA Oncol. 2016;2(6):823826. doi: 10.1001/jamaoncol.2015.6587

15. Wittekindt C, Wagner S, Sharma SJ, et al. HPV - A different view on Head and Neck Cancer. Laryngorhinootologie. 2018;97(S 01):S48-S113. doi: 10.1055/s-0043-121596

16. Stratton KL, Culkin DJ. A Contemporary Review of HPV and Penile Cancer. Oncology (Williston Park). 2016;30(3):245-249.

17. Berman TA, Schiller JT. Human papillomavirus in cervical cancer and oropharyngeal cancer: One cause, two diseases. Cancer. 2017;123(12):2219-2229. doi: 10.1002/cncr.30588

18. Ilboudo M, Zohoncon TM, Traore IMA, et al. Implication of Iow risk human papillomaviruses, HPV6 and HPV11 in laryngeal papillomatosis in Burkina Faso. Am J Otolaryngol. 2019;40(3):368-371. doi: 10.1016/j.amjoto.2019.02.003

19. National Cancer Institute. The Surveillance, Epidemiology, and End Results (SEER). Available online: https://seer.cancer.gov/statistics. Accessed on March 30, 2021.

20. World Health Organization International Agency for Research on Cancer. Global Cancer Observatory. Available online: https://gco. iarc.fr. Accessed on March 30, 2021.

21. Van Dyne EA, Henley SJ, Saraiya M, Thomas CC, Markowitz LE, Benard VB. Trends in Human Papillomavirus-Associated Cancers United States, 1999-2015. MMWR Morb Mortal Wkly Rep. 2018;67(33):918-924. doi: 10.15585/mmwr.mm6733a2

22. World Health Organization. Recomendations for routine immunization (updated: September 2020) Available online: http://www. who.int/immunization/policy/Immunization_routine table1.pdf Accessed on February 20, 2021.

23. Chan CK, Aimagambetova G, Ukybassova T, Kongrtay K, Azizan A Human Papillomavirus Infection and Cervical Cancer: Epidemiology, Screening, and Vaccination-Review of Current Perspectives. J Oncol. 2019;2019:3257939. doi: 10.1155/2019/3257939

24. Schiller J, Lowy D. Explanations for the high potency of HPV prophylactic vaccines. Vaccine. 2018;36(32 Pt A):4768-4773. doi: 10.1016/j.vaccine.2017.12.079

25. Stanley M, Dull P. HPV single-dose vaccination: Impact potential, evidence base and further evaluation. Vaccine. 2018;36(32 Pt A):4759-4760. doi: 10.1016/j.vaccine.2018.02.076

26. Schiller JT, Castellsagué X, Garland SM. A review of clinical trials of human papillomavirus prophylactic vaccines. Vaccine. 2012;30 Suppl 5(0 5):F123-F138. doi: 10.1016/j.vaccine.2012.04.108

27. Handler NS, Handler MZ, Majewski S, Schwartz RA. Human papillomavirus vaccine trials and tribulations: Vaccine efficacy. J Am Acad Dermatol. 2015;73(5):759-768. doi: 10.1016/j.jaad.2015.05.041 28. Joura EA, Giuliano AR, Iversen OE, et al. A 9-valent HPV vaccine against infection and intraepithelial neoplasia in women. $N$ Engl J Med. 2015;372(8):711-723. doi: 10.1056/NEJMoa1405044

29. Vesikari T, Brodszki N, van Damme P, et al. A Randomized, Double-Blind, Phase III Study of the Immunogenicity and Safety of a 9-Valent Human Papillomavirus L1 Virus-Like Particle Vaccine (V503) Versus Gardasil® in 9-15-Year-Old Girls. Pediatr Infect Dis J. 2015;34(9):992-998. doi: 10.1097/INF.0000000000000773

30. Nyg rd M, Saah A, Munk C, et al. Evaluation of the Long-Term Anti-Human Papillomavirus 6 (HPV6), 11, 16, and 18 Immune Responses Generated by the Quadrivalent HPV Vaccine. Clin Vaccine Immunol. 2015;22(8):943-948. doi: 10.1128/CVI.00133-15

31. Brotherton JML. Impact of HPV vaccination: Achievements and future challenges. Papillomavirus Res. 2019;7:138-140. doi: 10.1016/j.pvr.2019.04.004

32. Резолюция совета экспертов "Папилломавирусная инфекция: обзор накопленного опыта в решении мультидисциплинарной проблемы" // Эпидемиология и Вакцинопрофилактика. 2018. - T. 17. - № 6. - C. 109-113. [Expert Council Resolution
"Human Papillomavirus Infection: a Review of Experience in Solving a Multidisciplinary Problem". Epidemiology and Vaccinal Prevention. 2018;17(6):109-113. (In Russ).]

33. Tanaka H, Shirasawa H, Shimizu D, et al. Preventive effect of human papillomavirus vaccination on the development of uterine cervical lesions in young Japanese women. J Obstet Gynaecol Res. 2017;43(10):1597-1601. doi: 10.1111/jog.13419

34. World Health Organization. Progress and Challenges with Achieving Universal Immunization Coverage 2019 (data as July 2020). Available online: https://www.who.int/immunization/monitoring_surveillance/who-immuniz.pdf. Accessed on February 20, 2021

35. Sheikh S, Biundo E, Courcier S, et al. A report on the status of vaccination in Europe [published correction appears in Vaccine. 2019 Feb 28;37(10):1374-1376]. Vaccine. 2018;36(33):49794992. doi: 10.1016/j.vaccine.2018.06.044

36. Toh ZQ, Russell FM, Garland SM, Mulholland EK, Patton G, Licciardi PV. Human Papillomavirus Vaccination After COVID-19. JNCI Cancer Spectr. 2021;5(2):pkab011. doi: 10.1093/jncics/pkab011 37. Gavi. Overview of COVID-19 Situation in GAVI-Supported Countries and GAVI's Response 30th June 2020. Available online: https://www.gavi.org/vaccineswork/30-june-2020-overview-covid19-situation-gavi-supported-countries-gavi-response. Accessed on March 30, 2021

38. Девятилова А.Н. Возможности профилактики и ранней диагностики рака шейки матки на региональном уровне // Смоленский медицинский альманах. - 2019. - № 1. C. 95-97. [Devyatilova AN. Possibilities of prevention and early diagnosis of cervical cancer at the regional level. Smolensk medical almanac. 2019;(1):95-97. (In Russ).]

39. Симаходский А.С., Ипполитова М.Ф. Опыт формирования и реализации региональной программы иммунизации детского населения Санкт-Петербурга против папилломавирусной инфекции // Эпидемиология и Вакцинопрофилактика. 2019. - T. 18. - № 3. - C. 65-70. [Simakhodsky AS, Ippolitova MF. Experience of Formation and Implementation of the Regional Program of Immunization of Children's Population of St. Petersburg against Papillomavirus Infection. Epidemiology and Vaccinal Prevention. 2019;18(3):65-70. (In Russ).] doi: 10.31631/2073-3046-2019-18-3-65-70

40. Филиппов О.В., Большакова Л.Н., Елагина Т.Н., Новикова Ю.Б., Шаповалова Р.Ф., Аристова А.М. Региональный календарь профилактических прививок в Москве: история, развитие, перспективы // Эпидемиология и Вакцинопрофилактика. - 2020. T. 19. - № 4. - C. 63-75. [Filippov OV, Bolshakova LN, Elagina TN, Novikova YuB., Shapovalova RF, Aristova AM. Regional Schedule of Vaccination in Moscow: History, Development, Prospects. Epidemiology and Vaccinal Prevention. 2020;19(4):63-75. (In Russ).] doi: 10.31631/2073-3046-2020-19-4-63-75

41. Williams WW, Lu PJ, O'Halloran A, et al. Surveillance of Vaccination Coverage among Adult Populations - United States, 2015. MMWR Surveill Summ. 2017;66(11):1-28. doi: 10.15585/mmwr.ss6611a1

42. Skufca J, Ollgren J, Artama M, Ruokokoski E, Nohynek H, Palmu AA. The association of adverse events with bivalent human papilloma virus vaccination: A nationwide register-based cohort study in Finland. Vaccine. 2018;36(39):5926-5933. doi: 10.1016/j.vaccine.2018.06.074

43. Public Health England. Human papillomavirus (HPV) vaccination coverage in adolescent females in England: 2018/19: Report for England. Avaliable online: https://assets.publishing.service. gov.uk/government/uploads/system/uploads/attachment_data/ file/851797/HPV_annual_coverage_report_2018_to_2019.pdf. Accessed on March 30, 2021.

44. Walker TY, Elam-Evans LD, Yankey D, et al. National, Regional, State, and Selected Local Area Vaccination Coverage Among Adolescents Aged 13-17 Years - United States, 2018. MMWR Morb Mortal Wkly Rep. 2019;68(33):718-723. doi: 10.15585/mmwr.mm6833a2

45. Bogaards JA, Wallinga J, Brakenhoff RH, Meijer CJ, Berkhof J. Direct benefit of vaccinating boys along with girls against oncogenic human papillomavirus: bayesian evidence synthesis. BMJ. 2015;350:h2016. doi: 10.1136/bmj.h2016

46. Vänskä S, Luostarinen T, Baussano I, et al. Vaccination With Moderate Coverage Eradicates Oncogenic Human Papillomaviruses If 
a Gender-Neutral Strategy Is Applied. J Infect Dis. 2020;222(6):948956. doi: 10.1093/infdis/jiaa099

47. Faust $H$, Toft L, Sehr P, et al. Human Papillomavirus neutralizing and cross-reactive antibodies induced in HIV-positive subjects after vaccination with quadrivalent and bivalent HPV vaccines. Vaccine. 2016;34(13):1559-1565. doi: 10.1016/j.vaccine.2016.02.019

48. Lehtinen M, Luostarinen T, Vänskä S, et al. Gender-neutral vaccination provides improved control of human papillomavirus types 18/31/33/35 through herd immunity: Results of a community randomized trial (III). Int J Cancer. 2018;143(9):2299-2310. doi: 10.1002/ijc.31618

49. Drolet M, Bénard É, Boily MC, et al. Population-level impact and herd effects following human papillomavirus vaccination programmes: a systematic review and meta-analysis. Lancet Infect Dis. 2015;15(5):565-580. doi: 10.1016/S1473-3099(14)71073-4

50. Cameron RL, Kavanagh K, Pan J, et al. Human Papillomavirus Prevalence and Herd Immunity after Introduction of Vaccination Program, Scotland, 2009-2013. Emerg Infect Dis. 2016;22(1):5664. doi: 10.3201/eid2201.150736

51. Brisson M, Bénard É, Drolet M, et al. Population-level impact, herd immunity, and elimination after human papillomavirus vaccination: a systematic review and meta-analysis of predictions from transmission-dynamic models. Lancet Public Health. 2016;1(1):e8e17. doi: 10.1016/S2468-2667(16)30001-9

52. Elfström KM, Lazzarato F, Franceschi S, Dillner J, Baussano I. Human Papillomavirus Vaccination of Boys and Extended Catchup Vaccination: Effects on the Resilience of Programs. J Infect Dis. 2016;213(2):199-205. doi: 10.1093/infdis/jiv368
53. Chow EPF, Machalek DA, Tabrizi SN, et al. Quadrivalent vaccine-targeted human papillomavirus genotypes in heterosexual men after the Australian female human papillomavirus vaccination programme: a retrospective observational study [published correction appears in Lancet Infect Dis. 2017 Jan;17 (1):17]. Lancet Infect Dis. 2017;17(1):68-77. doi: 10.1016/S1473-3099(16)30116-5

54. Spinner C, Ding L, Bernstein DI, et al. Human Papillomavirus Vaccine Effectiveness and Herd Protection in Young Women. Pediatrics. 2019;143(2):e20181902. doi: 10.1542/peds.2018-1902

55. Machalek DA, Chow EP, Garland SM, et al. Human Papillomavirus Prevalence in Unvaccinated Heterosexual Men After a National Female Vaccination Program. J Infect Dis. 2017;215(2):202-208. doi: 10.1093/infdis/jiw530

56. Kavanagh K, Pollock KG, Cuschieri K, et al. Changes in the prevalence of human papillomavirus following a national bivalent human papillomavirus vaccination programme in Scotland: a 7 -year cross-sectional study. Lancet Infect Dis. 2017;17(12):1293-1302. doi: 10.1016/S1473-3099(17)30468-1

57. Novakovic D, Cheng ATL, Zurynski Y, et al. A Prospective Study of the Incidence of Juvenile-Onset Recurrent Respiratory Papillomatosis After Implementation of a National HPV Vaccination Program. J Infect Dis. 2018;217(2):208-212. doi: 10.1093/infdis/jix498

58. Lehtinen M, Söderlund-Strand A, Vänskä S, et al. Impact of gender-neutral or girls-only vaccination against human papillomavirus-Results of a community-randomized clinical trial (I). Int J Cancer. 2018;142(5):949-958. doi: 10.1002/ijc.31119

\section{ИНФОРМАЦИЯ ОБ АВТОРАХ}

Суровцева Ольга Викторовна [Olga V. Surovtseva], адрес: Россия, 117997, Москва, ул. ул. Островитянова, д. 1 [address: 1 Ostrovityanova street, 117997, Moscow, Russia]; телефон: 8(905) 190-79-74; e-mail: dr.surovtseva@yandex.ru

Привалова Татьяна Евгеньевна, к.м.н. [Tatiana E. Privalova, MD, PhD], адрес: Россия, 119333, Москва, ул. Фотиевой, д. 10 [address: 10 Fotievoy street, 119333, Moscow, Russia]; телефон: 8 (499) 400-47-33; eLibrary SPIN: 7879-4299

Андриянов Дмитрий Валериевич [Dmitry V. Andriyanov], адрес: Россия, 117997, Москва, ул. Островитянова, д. 1 [address: 1 Ostrovityanova street, 117997, Moscow, Russia]; e-mail: Andriynov-Dima@yandex.ru 